Revue internationale P.M.E.

Économie et gestion de la petite et moyenne entreprise

\title{
Différence de comportements et réciprocité d'intérêts dans le partenariat entre entreprises de pays d'inégal développement
}

\section{Khaled Gherzouli}

Volume 10, numéro 1, 1997

URI : https://id.erudit.org/iderudit/1009018ar

DOI : https://doi.org/10.7202/1009018ar

Aller au sommaire du numéro

Éditeur(s)

Presses de l’Université du Québec

ISSN

0776-5436 (imprimé)

1918-9699 (numérique)

Découvrir la revue

Citer cet article

Gherzouli, K. (1997). Différence de comportements et réciprocité d'intérêts dans le partenariat entre entreprises de pays d'inégal développement. Revue internationale P.M.E., 10(1), 73-102. https://doi.org/10.7202/1009018ar
Résumé de l'article

Le partenariat interentreprises Nord-Sud implique des comportements différenciés. Les facteurs de motivation et l'engagement de chaque partie sont rarement similaires. Aussi, pour dépasser cette asymétrie de projet, la présente contribution met en exergue le rôle important d'une réciprocité large d'intérêts. Les partenaires doivent, en effet, concevoir leur accord comme une symbiose organisée de codéveloppement qui passe par l'élargissement des réciprocités de base (celles établies à l'origine de l'accord) et la recherche continue d'espaces coopératifs plus féconds. L'article propose également une réflexion sur la nature de l'engagement à respecter pour aboutir à de telles conduites coopératives. 


\title{
Différence de comportements et réciprocité d'intérêts dans le partenariat entre entreprises de pays d'inégal développement
}

Khaled GHERZOULI*

Université de Rouen

\section{MOTS CLÉS}

\section{Partenariat interentreprises - Coopération Nord-Sud \\ Réciprocité d'intérêts - Asymétrie de projet \\ Dynamique du partenariat - PME - Groupe industriel}

\begin{abstract}
RÉSUMÉ
Le partenariat interentreprises Nord-Sud implique des comportements différenciés. Les facteurs de motivation et l'engagement de chaque partie sont rarement similaires. Aussi, pour dépasser cette asymétrie de projet, la présente contribution met en exergue le rôle important d'une réciprocité large d'intérêts. Les partenaires doivent, en effet, concevoir leur accord comme une symbiose organisée de codéveloppement qui passe par l'élargissement des réciprocités de base (celles établies à l'origine de l'accord) et la recherche continue d'espaces coopératifs plus féconds. L'article propose également une réflexion sur la nature de l'engagement à respecter pour aboutir à de telles conduites coopératives.
\end{abstract}

* Nous remercions les trois lecteurs anonymes pour leurs remarques et suggestions constructives pour les deux premières versions de cette contribution. 


\begin{abstract}
The North-South interfirms partnership entails various behaviours. The motivations and the involvements of each partner are rarely similar. Thus, with the purpose of overcoming this asymmetry of project, this article puts forward the important role of a large reciprocity of interests. Indeed, the partners have to conceive their agreement as an organized symbiosis of co-development which goes through the extension of basic reciprocities and the continuous definition of new combinations of activities which are favourable to all. This contribution also suggests a reflection on the nature of the involvement which is to be respected so as to reach such cooperative attitudes.
\end{abstract}

\title{
RESUMEN
}

La colaboración interempresas Norte-Sur implica comportamientos distintos. Las motivaciones y el empeño de cada parte son raramente similares. Por eso, para rebasar esta asimetria de proyectos, ésta contribución recalca la necesidad que existe una reciprocidad de intereses grande. En effecto, los participantes deben concebir estos acuerdos como una simbiosis organizada de co-desarrollo que pasa por la ampliación de las reciprocidades bàsicas y la bùsqueda continua de espacios cooperativos màs fecundos. El articulo propone también una reflexiòn sobre la naturaleza del empeño que se debe respetar para incitar tales conductas cooperativas.

\section{Introduction}

Dans un monde de forte complexité, les interactions entre États, tout comme celles entre les entreprises, sont de plus en plus variées et déterminantes. Elles se révèlent aussi bien dans des processus d'échanges commerciaux de plus en plus divers, que dans des conduites interentreprises multiformes. Entre le Nord et le Sud, cette interdépendance ne cesse de s'exacerber pour enfanter de nouvelles situations de coopération.

\section{L'AUTEUR}

Khaled Gherzouli détient un doctorat en sciences de gestion et est maître de conférences à l'Université de Rouen (CREGO de Dijon). Ses travaux portent sur les aspects stratégiques et organisationnels des relations partenariales entre entreprises de pays à développement inégal. Adresse : 04 ter, rue Pillore, 76000 Rouen, France. 
Certes, la coopération entre pays développés et pays en développement est loin de constituer un phénomène nouveau ${ }^{1}$. Dénommée souvent relation de transfert de technologie, elle remonte même au lendemain de l'indépendance des pays du Sud. Toutefois, de multiples bouleversements politico-économiques récents sont venus modifier l'établissement et la conduite de ce type d'accords. En effet, les États du Nord commencent à s'intéresser de plus en plus au devenir des pays du Sud et, notamment, à la croissance de ceux situés au sein de leur zone d'influence ${ }^{2}$. Par ailleurs, les phénomènes de mondialisation des marchés et de délocalisation de certaines activités obligent davantage d'entreprises occidentales à chercher de meilleures relations avec leurs partenaires du Sud. Devant la complexité croissante des technologies, les firmes du Sud ne cessent de leur côté de nouer des rapports renforcés avec leurs fournisseurs du Nord.

Il est possible, somme toute, de remarquer que ces relations cessent d'être, dans bien des cas, de simples accords de transferts de technologie pour se transformer en opérations de partenariat (CNUCED, 1990). La pratique de contrats «clés en main» ou "produit en main », largement préférée dans le passé, est de plus en plus écartée. La coopération désormais souhaitée exige la mise en place d'accords de coopération entre entreprises indépendantes qui s'engagent mutuellement, dans une perspective de longue durée, pour atteindre une multitude d'objectifs. Le partenariat interentreprises doit être ainsi envisagé comme un accord amélioré, dans la mesure où il implique l'engagement durable et risqué des parties prenantes et l'attachement de celles-ci aux résultats susceptibles d'être obtenus par le projet mené conjointement.

C'est ce type d'accords interentreprises que nous voulons examiner. À travers l'étude de trois cas concrets de partenariat, nous nous intéresserons particulièrement aux rapprochements entre grandes entreprises occidentales et PME du Sud. Nous constaterons ainsi que ce type d'accords est loin de refléter systématiquement un programme de coopération pure. Le partenariat interentreprises reste en réalité un «conflit-coopération» qui, selon la façon dont

1. En matière de coopération et depuis fort longtemps, les pays du Nord et ceux du Sud empruntent en général trois voies différentes : la coopération d'État à État, la coopération orchestrée par l'intermédiaire de différents organismes internationaux et la coopération mettant en lien des entreprises des deux zones.

2. Ainsi, le Japon ne cesse d'entretenir des relations privilégiées avec les pays de sa périphérie géographique (Asie du Sud-Est) pour sauvegarder la dynamique du développement largement amorcée dans ces pays et tirer parti de leur croissance accélérée. Les États-Unis et le Canada travaillent également dans le même sens avec les pays d'Amérique latine, notamment dans le cadre de l'ALENA. De son côté, l'Union européenne n'échappe pas à cette logique ; maints programmes de coopération sont établis avec les pays d'Europe centrale et orientale ainsi qu'avec les pays de la rive sud méditerranéenne. 
il est mené et la logique stratégique avec laquelle il est poursuivi, peut se transformer en partenariat coopératif (toutes les parties sont gagnantes) ou en partenariat opportuniste (un seul gagnant).

Considérer que toutes les relations ne relèvent pas nécessairement du partenariat coopératif conduit à rechercher les critères de pertinence, c'est-à-dire les facteurs à satisfaire pour qu'une démarche partenariale positive soit possible. Dans cette optique, il est important de centrer l'étude sur les unités actives du partenariat (les entreprises partenaires) pour comprendre la logique stratégique de leur comportement. Le but est de montrer qu'au-delà des asymétries de comportements possibles, l'élément le plus critique dans ces rapprochements est lié à la nature des réciprocités d'intérêts établies et la portée de l'engagement assumé.

\section{Comportements stratégiques différenciés dans le partenariat Nord-Sud}

Dans cette contribution, l'élément qui retient notre attention est la logique stratégique qui guide les partenaires devant s'impliquer dans ce type de liaison. La conduite des arrangements interentreprises dans un contexte Nord-Sud dépend certes de maints facteurs (politico-économiques, socioculturels, organisationnels, etc.). Toutefois, seuls les aspects stratégiques seront examinés de près dans cette étude, et ce, pour mieux se centrer sur le caractère dynamique de ces accords eu égard aux comportements différenciés des partenaires impliqués.

Dans cette perspective, il est très utile de retenir l'approche stratégique de L. Poirier (1987) qui distingue la stratégie à but positif et la stratégie à but négatif. La première est une stratégie offensive : elle cherche à poser l'entreprise comme entité voulant concrétiser son projet. La seconde, en revanche, est une stratégie défensive : elle manifeste le désir de l'entreprise de ne pas être réduite à la passivité. En rappelant, par ailleurs, que la stratégie est à la fois contenu et processus, on peut donc examiner les accords de partenariat à travers deux critères essentiels : d'un côté, il faut considérer l'aspect offensif ou défensif de la motivation au partenariat; de l'autre, il faut traiter la portée du risque assumé et l'aspect actif ou passif de cet engagement.

\subsection{Motivation offensive contre motivation défensive}

Dans les facteurs de motivation conduisant les firmes à envisager des accords de partenariat, il faut distinguer, d'une part, les cas où ces accords sont conclus pour résoudre des difficultés ou réduire des contraintes et, d'autre part, les partenariats offensifs fondés sur des aspects de développement et une volonté de croissance. 


\subsubsection{Partenariat offensif}

L'aspect volontariste du partenariat se présente dès l'instant où celui-ci est vu comme une nécessité stratégique; il est une «action» en faveur du développement de l'entreprise. Les arguments offensifs en faveur du partenariat sont très variés, étant donné l'asymétrie des acteurs présents et le point de vue examiné : grandes firmes du Nord ou PME du Sud.

Dans l'optique de la grande firme occidentale, le partenariat se comprend davantage avec l'objectif avoué de rechercher des débouchés permanents sur des marchés réputés réglementés et lointains, au moins par le relais d'un partenaire local (Schillaci, 1987). Il est à rappeler par ailleurs que le partenariat, comme stratégie de pénétration de marchés et de développement international, ne s'appuie pas seulement sur l'axe produit. Plusieurs entreprises font au contraire de la valorisation de leur potentiel technologique l'axe privilégié de leur politique de coopération. Le souci d'exploiter des avantages techniques est souvent évoqué comme une seconde motivation au partenariat après la pénétration de nouveaux marchés (Snoy, 1988).

Le partenariat offensif est aussi présent dans les stratégies que mènent certaines entreprises pour s'assurer un approvisionnement sans faille en ressources naturelles. Cette possibilité est très caractéristique des projets de partenariat dans le domaine pétrolier et minier. Par ailleurs, et dans une logique légèrement différente, le «partenariat action » peut également servir une politique de rationalisation de la production par délocalisation pour bénéficier de l'abondance des ressources naturelles et du faible coût de la main-d'œuvre dans les pays en développement. Cette explication est, par exemple, à l'origine de la création de multiples entreprises conjointes dans les activités manufacturières à faible valeur ajoutée, comme la chaussure et la confection, entre firmes japonaises et firmes de pays d'Asie du Sud-Est (Ozawa, 1989).

Du point de vue de la PME du Sud, l'action de partenariat peut être une simple démarche de valorisation d'un atout commercial, un moyen de dépassement de l'activité de distribution pour amorcer ses premiers pas dans la production ou, enfin, une stratégie appropriée en vue d'obtenir un apprentissage technologique accéléré. Le mobile " apprentissage " se rencontre à l'échelle d'entreprises dotées déjà d'une certaine culture industrielle. Il s'agit de firmes qui n'hésitent pas à appréhender le partenariat comme une interrelation favorable à l'émergence d'apprentissages aussi bien techniques que managériaux (Gherzouli, 1995). L'objectif ultime est l'acquisition et l'assimilation de certaines technologies. C'est également le cas de PME ayant parfaitement saisi l'urgence de certaines mises au point techniques pour atteindre les qualités technologiques recherchées par leurs clients, et qui ne tardent pas à envisager des projets de partenariat pour améliorer leurs productions (mieux répondre aux exigences 
de plus en plus complexes des consommateurs locaux, développement des moyens de télécommunication oblige).

Parce qu'elles se sentent de plus en plus menacées par la concurrence, certaines entreprises du Sud (pays du Sud-Est asiatique) s'attachent à modifier leur insertion dans la division internationale du travail en bénéficiant des conditions de production locales toujours plus économiques. Il s'agit concrètement pour celles-ci de chercher, par le biais du partenariat, à réussir une augmentation sensible du contenu technologique (plus de valeur ajoutée) des industries qu'elles développent (Ernst et O'Connor, 1989).

Le partenariat peut aussi se concevoir dans le cadre d'une stratégie d'intégration globale. Celle-ci peut intervenir en amont lorsque, par exemple, l'entreprise développe à travers un projet de partenariat la fabrication d'un sousproduit ou d'un composant nécessaire à sa production principale. Elle peut en revanche s'exercer en aval quand le but est de renforcer le contrôle qualité, afin d'explorer l'exportation vers des marchés internationaux (Lall, 1990).

Une autre pratique peut être également distinguée. Elle concerne les PME commerciales qui décident d'engager des projets de partenariat avec, comme approche, la mise en œuvre d'unités de montage. Cette situation se rencontre aussi chez des individus qui ont acquis une expérience non négligeable, soit à l'étranger où ils ont travaillé et développé une maîtrise technique appréciable, soit au niveau national auprès d'entreprises publiques dans lesquelles ils avaient des responsabilités. Ils recourent alors au partenariat pour mettre en place de petits projets de production que l'on peut identifier comme de simples unités de montage (Lall, 1990).

Le tableau qui suit récapitule les principaux facteurs de motivation à l'origine du partenariat offensif dans les conduites concertées Nord-Sud.

TABLEAU 1

Facteurs de motivation à l'origine du partenariat offensif

\begin{tabular}{ll}
\hline \multicolumn{1}{c}{ Entreprises du Nord } & \multicolumn{1}{c}{ Entreprises du Sud } \\
\hline - S'assurer de nouveaux débouchés et & - Valoriser un atout commercial. \\
prendre place sur des marchés réputés & - Dépasser l'activité de distribution pour \\
difficiles, réglementés et lointains. & amorcer ses premiers pas dans la production. \\
$-\quad$ Valoriser son potentiel technologique. & - Acquérir de nouvelles technologies et asseoir \\
- S'assurer un approvisionnement sans & une meilleure maîtrise de celles-ci. \\
faille en ressources naturelles. & - Servir une stratégie d'intégration. \\
- Servir une stratégie de rationalisation & - Atteindre les qualités technologiques \\
de la production. & requises, notamment à l'échelle des marchés \\
& à l'exportation. \\
\hline
\end{tabular}




\subsubsection{Partenariat défensif}

L'aspect défensif dans le partenariat est présent lorsque la motivation principale amenant l'entreprise dans cette voie est la volonté de contrer une menace. L'entreprise, devant une nouvelle situation de contrainte, décide alors de changer de manœuvre afin de l'éviter.

Au niveau du partenaire du Nord, la logique défensive des accords se présente sous plusieurs facettes. En premier lieu, il peut s'agir de l'exigence d'une plus grande répartition des risques inhérents à l'implantation sur les marchés étrangers, par la diversification de débouchés. Cela permet non seulement de compenser des insuccès éventuels, mais surtout de faire face à des événements politiques qui accentuent les risques d'opérations effectuées dans certains milieux économiques du Sud (lourdeur bureaucratique, restrictions excessives sur les rémunérations des services rendus, restrictions frappant l'achat de biens d'équipements, etc.) [Contractor et Lorange, 1988]. Il importe de souligner que ces obstacles réglementaires furent une des principales causes de l'accroissement des actions de coopération au milieu des années 1970 (Oman, 1984).

Une autre motivation puissante au partenariat, dans un monde de concurrence exacerbée, est la nécessité de trouver des débouchés externes à des activités qui, sur le marché national, ne garantissent plus une croissance suffisante. Une enquête sur le partenariat des entreprises européennes avec celles des pays d'Amérique latine révèle que les firmes du Nord s'expatrient dès que leur marché intérieur arrive au point de saturation (CNUCED, 1984). D'autres entreprises utilisent les projets de partenariat pour exécuter leurs stratégies de désinvestissement pour des produits ou des marchés arrivés à maturité (Oman, 1989).

Depuis le début des années 1980, la mondialisation des marchés ne cesse de se manifester sous l'effet conjugué de l'ouverture des frontières, de la rapidité des changements technologiques et du développement extraordinaire des moyens de télécommunication. Devant ce nouveau contexte, les grandes entreprises occidentales ont tendance à poursuivre deux objectifs complémentaires: rechercher une compétitivité accrue qui passe désormais par une stratégie globale appréhendant le monde comme un seul marché, et mettre sur pied des réseaux de relations internationales. Aussi, dans certaines activités, il devient de plus en plus nécessaire, pour mieux servir ces stratégies de globalisation, d'opérer des actions de partenariat même avec des entreprises de pays dits «complémentaires de la Triade». Le partenariat est, en ce sens, une réaction de défense à l'égard des pressions qu'exercent les cohérences interentreprises dans le cadre de certains marchés mondiaux. 
La détérioration des dotations en facteurs de production constitue une autre explication du comportement partenarial. Pour éviter de délocaliser complètement leurs activités vers les pays où main-d'œuvre et matières premières sont plus abondantes, d'innombrables entreprises choisissent la coopération interentreprises comme solution de rechange. L'effort en vue d'une plus grande efficacité sur le plan des coûts figure en bonne place parmi les diverses motivations que soulignent les entreprises pour expliquer leurs actions de partenariat (APRODI, 1989).

Du côté des partenaires du Sud, il est fréquent de souligner que ceux-ci s'engagent dans des actions de partenariat pour des raisons à caractère offensif. Cela ne signifie pas pour autant que les PME du Sud échappent à la logique défensive.

D'une part, il est question d'un nombre de firmes qui, faisant face à des problèmes de renouvellement de procédés techniques de fabrication et $n$ 'ayant ni les ressources financières suffisantes ni le savoir-faire indispensable, se sont lancées dans des actions de collaboration pour redéfinir leurs compétences techniques et mieux asseoir leur productivité. Dans les pays du Sud, nombreuses sont les firmes qui disposent d'un outil industriel relativement performant, mais qui reste imparfaitement exploité faute de pièces de rechange ou de révisions techniques nécessaires. Ces entreprises ne tardent pas alors à envisager des actions de partenariat pour aménager les installations existantes et améliorer le fonctionnement (Gherzouli, 1995). Il est à souligner que cette attitude est fréquente, notamment, chez les entreprises à culture industrielle.

Il convient d'évoquer, d'autre part, le partenariat rendu obligatoire par une conjoncture macro-économique particulière. C'est le cas, par exemple, de partenariats envisagés pour bénéficier des lignes de crédits accordées par les gouvernements occidentaux. L'endettement massif des pays du Sud, qui limite fortement leurs possibilités d'importation de produits et encore plus d'usines clés en main, comme la pratique s'en était répandue dans les années 1970, encourage les entreprises de ces pays à envisager des actions concertées pour pouvoir obtenir des fabrications locales au prix d'un investissement moins lourd.

Devant l'urgence d'envisager l'exportation, problème d'accès à la devise oblige, certaines firmes souhaitent développer des relations de partenariat afin d'accéder aux marchés internationaux. En ce sens, le partenariat est une réaction adaptée pour tenter de résoudre les problèmes que pose l'exportation notamment sur les marchés de l'Occident, que la PME du Sud n'a que peu de chances de conquérir seule (Durand, 1995). Il n'est pas rare, cependant, que ce type de partenariat soit envisagé dans une perspective de développement et de croissance. Il s'agira alors plus d'un partenariat offensif que d'une réaction de défense. 
En somme, il importe de regrouper les principales motivations à la source du partenariat défensif de la manière qui suit.

TABLEAU 2

Facteurs de motivation essentiels à l'origine du partenariat défensif

Entreprises du Nord

- Mieux répartir les risques inhérents à la présence sur les marchés du Sud.

- Contourner certaines restrictions législatives.

- Échapper aux entraves à la croissance au sein des marchés du Nord.

- Faire face au renchérissement de la main-d'œuvre dans les pays développés.

- Servir une stratégie de retrait progressif.
Entreprises du Sud

- Renouveler les procédés techniques utilisés.

- Aménager les installations existantes et améliorer le fonctionnement.

- Négocier les possibilités de financement par lignes de crédits accordés par les pouvoirs publics des partenaires du Nord.

- Contourner les obstacles à l'exportation sur les marchés occidentaux.

S'interroger sur les principales préoccupations des protagonistes dans le partenariat permet donc de souligner une multiplicité de facteurs de motivation dans ce type de coalition. Il convient cependant de remarquer une importante différence entre les mobiles des partenaires du Nord et ceux des firmes du Sud. Quel que soit le contexte de départ (urgence de faire face à une contrainte ou volonté offensive de croissance), les grandes firmes occidentales semblent tout compte fait intéressées uniquement par des aspects commerciaux. L'objectif ultime est de s'assurer régulièrement un volume d'affaires conséquent. Pour les PME du Sud, les aspects commerciaux sont certes pris en compte, mais ils sont accessoires au regard de l'appropriation des techniques et de l'accroissement de l'efficacité de leur potentiel productif.

En conséquence, il est clair que la réussite partenariale recherchée par les partenaires du Nord est divergente de celle que prônent les partenaires du Sud. Les premiers privilégient le succès commercial (importance du chiffre d'affaires réalisé, nombre de contrats de fourniture de composants conclus et diversité des prestations immatérielles fournies), alors que les seconds visent une réussite plus globale ${ }^{3}$, dépassant le simple partage d'opportunités de marchés et impliquant, en dehors des gains pécuniaires possibles, la mise en œuvre d'un réel processus de production, l'assimilation de nouvelles technologies, l'apprentissage de nouvelles méthodes d'organisation et la rationalisation de l'outil de production existant.

3. Il convient de signaler que cette réussite n'est recherchée que par les entreprises dotées d'une certaine culture industrielle. Il existe en effet un nombre important de PME du Sud qui n'envisagent le partenariat que dans une optique purement commerciale. 
Pour conclure, précisons que la distinction entre partenariat offensif et partenariat défensif n'est pas aussi catégorique qu'elle paraît. En effet, il n'est pas rare de relever, dans les motivations conduisant chaque firme au partenariat, des facteurs conjugués de contraintes à dépasser et d'intentions d'expansion et de croissance. Il en est ainsi lorsque ce type de conduite est favorisé dans le double but de trouver de nouveaux débouchés et de résoudre des problèmes de saturation du marché national. C'est également le cas lorsque l'objectif visé est, d'une part, la maîtrise technologique et l'amélioration de la qualité des produits offerts et, d'autre part, le dépassement des contraintes d'importations de composants et de matières premières. En somme, s'il est fort probable de rencontrer dans chaque comportement partenarial aussi bien des motivations défensives que des motivations offensives, il est néanmoins possible de distinguer des situations plus marquées par des aspects offensifs et d'autres plus caractérisées par des facteurs défensifs.

Le partenariat interentreprises Nord-Sud est donc l'occasion d'un affrontement entre deux orientations : la logique du partenaire occidental et celle de la partie en développement. Cette distinction des facteurs de motivation au partenariat nécessite, cependant, d'être complétée par une seconde en rapport avec la nature de l'engagement de chaque allié dans ce type de rapprochement. Cela nous conduit à considérer le critère dynamique ou passif de l'attitude de l'entrepreneur face au choix du partenariat.

\subsection{Implication active contre implication passive}

L'idée d'un éventuel partenariat peut venir de différentes personnes : des responsables opérationnels, de la direction générale ou du partenaire potentiel. Cependant, le partenariat, comme on l'a déjà souligné, peut selon l'implication des partenaires être actif ou passif.

Le partenariat est une manœuvre active si tout d'abord il est naturellement intégré dans la stratégie globale des parties. En effet, après avoir pris conscience qu'elles ne peuvent plus se contenter d'une réflexion stratégique classique (uniquement concurrentielle), les firmes aujourd'hui ne s'attardent plus à développer des pratiques concertées. La prise en compte des accords du point de vue de la stratégie des entreprises impose de ne pas les considérer comme objet unique, mais comme partie de leurs activités et donc élément soumis à leurs stratégies propres. Le partenariat peut, par exemple, se présenter comme une manœuvre au service d'un processus d'internationalisation, comme il peut être au service de toute autre stratégie (recentrage sur le métier principal, intégration, diversification, etc.).

Il va de soi que le partenariat réduit l'incertitude et permet le partage des risques entre partenaires. Toutefois, cela ne signifie pas absence totale de risques. 
Pour fonctionner et durer, le partenariat requiert l'engagement mutuel et résolu des partenaires. D'un premier point de vue, nous considérons que nous sommes en présence d'une implication risquée de la part des participants si les contributions de ceux-ci présentent une valeur élevée et lorsque le coût de l'engagement est important. Il faut entendre par «coût de l'engagement» l'ensemble des dépenses relatives à la mise en œuvre du projet de partenariat, depuis son élaboration initiale et la recherche d'un partenaire jusqu'à la mise au point tangible du projet.

La nature des apports réunis peut aussi nous renseigner sur la qualité de l'engagement des partenaires. L'engagement deviendra conséquent au fur et à mesure que les ressources apportées deviennent spécifiques. Une ressource est dite "spécifique» si elle ne peut pas être réutilisée à d'autres fonctions ou par d'autres utilisateurs sans perdre une partie de sa valeur productive (Williamson, 1975 et 1985). La spécificité des actifs ne se limite pas aux éléments physiques. Williamson en distingue cinq types: la spécificité spatiale ou l'immobilité de l'implantation géographique, la spécialisation fonctionnelle (c'est-à-dire l'affectation d'un actif à un usage précis), la compétence et le savoir-faire humain, le capital de renommée (la réputation) et la spécialisation par rapport au débouché (Everaer, 1993). Dans le partenariat Nord-Sud, cette spécificité des ressources peut être observée en particulier dans l'adaptation des technologies aux conditions particulières des pays en développement. Elle se présente aussi dans les programmes spécialisés de formation du personnel et dans la connaissance pointue de la pratique des affaires au regard de certains débouchés.

En dépit du fait qu'il soit offensif ou défensif, le partenariat ne peut être qualifié d'actif ou de dynamique que dans la mesure où il fait partie de la stratégie d'ensemble de la firme, et que s'il est mis en œuvre avec célérité et une réelle volonté d'engager les moyens requis en assumant les risques possibles.

La pratique du partenariat nous renseigne toutefois sur de nombreuses situations qui ne peuvent être identifiées comme des partenariats actifs. Il s'agit plutôt de partenariats passifs dont les caractéristiques sont tout à fait l'inverse de celles évoquées ci-dessus. L'implication recherchée est souvent la moindre possible. Elle se borne aux services les plus simples, les plus faciles à fournir et, vise notamment, ceux qui impliquent des objets plutôt que des personnes. Le partenariat est ici une simple occasion d'affaires qui n'influe guère sur la position concurrentielle de l'entreprise et reste le résultat du hasard (la recherche de cette occasion est loin d'être systématique). Dans ce type de présence, l'origine de l'initiative se trouve souvent à l'extérieur de la firme.

Le partenariat passif est, par conséquent, celui qui se reflète dans une action qui ne comporte qu'un minimum de risque ; il ne repose pas sur une préparation solide et ne s'intègre pas dans la logique globale de l'entreprise (tableau 3). 


\section{TABleau 3}

\section{L'implication dans le partenariat Nord-Sud}

Partenariat à implication active

- Engagement risqué (importance des ressources réunies et spécificité des actifs engagés).

- Implication continue dès le stade de la formulation progressive du projet jusqu'à sa réalisation définitive et son exploitation.

- Une action trouvant sa logique dans la stratégie d'ensemble que mène l'entreprise et qui fait partie intégrante du système de valeurs de celle-ci.
Partenariat à implication passive

- Engagement sans risque majeur.

- Opération réalisée dans la hâte sans un réel travail de préparation et de mise en œuvre.

- Opération sporadique sans lien direct avec les stratégies que mène la firme (une simple action de circonstance).

\subsection{Symétrie et asymétrie de projet dans le partenariat}

Il convient maintenant, à la lumière des deux critères évoqués (actif-passif, offensif-défensif), de dresser une distinction de quatre types de partenariat. En effet, l'attitude et la motivation de chaque partenaire dans cette conduite concertée peuvent prendre l'une des figures suivantes: offensif-actif, offensif-passif, défensif-actif, et défensif-passif. Les caractéristiques de chaque situation sont résumées dans le tableau ci-après.

\section{TABLEAU 4}

\section{Typologie du partenariat en termes de motivations et d'implications}

\begin{tabular}{|c|c|c|c|}
\hline & \multicolumn{2}{|c|}{ 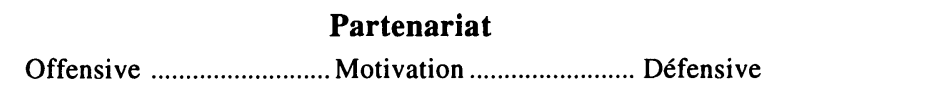 } \\
\hline \multirow{2}{*}{ 焉 } & $\begin{array}{c}\text { Active } \\
1 \\
1 \\
1 \\
1\end{array}$ & $\begin{array}{l}\text { Le partenariat s'inscrit ici dans la } \\
\text { stratégie d'ensemble de l'entreprise. } \\
\text { Il est en relation avec la volonté } \\
\text { d'exploiter des occasions ou des } \\
\text { situations favorables. }\end{array}$ & $\begin{array}{l}\text { Développer pour outrepasser des situations } \\
\text { de contraintes; le partenariat est volontaire, } \\
\text { planifié et pratiqué avec engagement. }\end{array}$ \\
\hline & $\begin{array}{c}\text { Implication } \\
1 \\
1 \\
\text { ' } \\
\text { Passive }\end{array}$ & $\begin{array}{l}\text { Issu d'une volonté de tirer profit } \\
\text { d'une circonstance opportune, il reste } \\
\text { une manœuvre secondaire par } \\
\text { rapport aux différentes actions que } \\
\text { mène la firme. L'implication est } \\
\text { sans risque majeur. }\end{array}$ & $\begin{array}{l}\text { Une manœuvre pour faire face à une contrainte } \\
\text { L'attitude reste cependant réservée et l'envie de } \\
\text { coopérer n'est que sporatique. }\end{array}$ \\
\hline
\end{tabular}

En somme, si l'on sait que l'engagement de tout partenaire peut prendre l'une des quatre figures évoquées ci-dessus, le croisement des différentes figures de chaque partie avec celles de son partenaire donne alors seize situations diverses, parmi lesquelles il est possible de distinguer les situations de partenariat asymétrique, les situations de partenariat symétrique actif et les situations de symétrie passive (figure 1). 
FIGURE 1

Symétrie et asymétrie de projet dans le partenariat

Comportement partenarial de la partie A

Comportement partenarial de la partie B

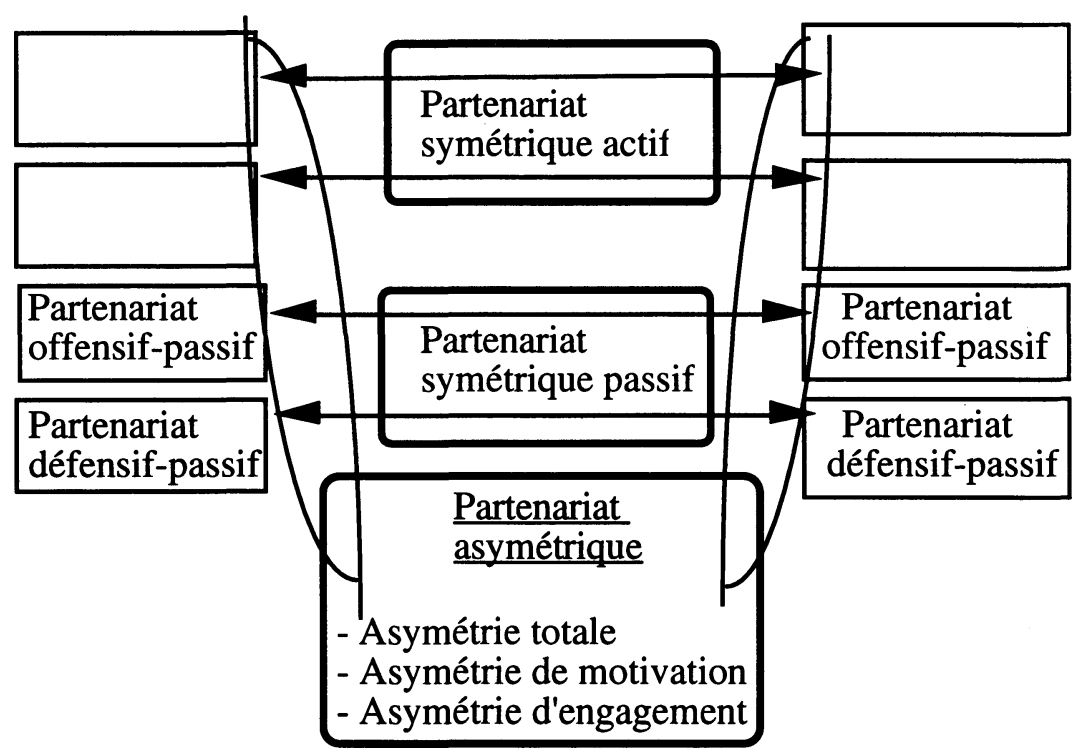

On relève, en somme, une prédominance des situations d'asymétrie de projet (12 sur 16). La probabilité que les partenaires se présentent avec des motivations divergentes et une implication dissemblable reste très forte. Les situations asymétriques peuvent être, par ailleurs, totales ou partielles. Elles sont totales lorsque l'asymétrie concerne aussi bien la motivation que l'engagement ; elles sont en revanche partielles quand l'asymétrie implique seulement l'un des deux aspects. On note ainsi la présence de cas d'asymétrie de motivation et de cas d'asymétrie d'engagement.

Pour les situations rares de symétrie de projet (même motivation et même engagement), il convient de considérer, d'une part, les cas de symétrie active qui sous-tendent que le partenariat est actif pour l'ensemble des partenaires impliqués et, d'autre part, les situations de symétrie passive qui sous-tendent que le partenariat est passif pour tous. Cette dernière posture ne représente pas en réalité une figure de partenariat. Elle ne peut déboucher que sur des résultats médiocres, pour cause de passivité de partenaires. Faut-il pour autant s'étonner si l'on sait que, par définition, le partenariat exige de prime abord une implication profonde et risquée de toutes les parties concernées. De leur côté, les 
cas de symétrie active ne reflètent pas une réelle situation de similitude de projet, étant donné la singularité des firmes présentes dans le partenariat Nord-Sud. En effet, les partenaires peuvent certes présenter le même type de motivation (offensive ou défensive) sans toutefois avoir les mêmes objectifs ; le partenariat ici étudié fait souvent intervenir des firmes dont les différentiels de niveaux technologiques et d'orientations stratégiques sont relativement importants.

Tout bien considéré, l'interprétation stratégique du partenariat Nord-Sud soulève une interrogation intéressante quant à la conduite de ce type de rapprochement interentreprises. L'existence de différences dans les comportements des partenaires, définis en termes d'implications et de motivations stratégiques, n'entraîne-t-elle pas des situations de crises ou d'échecs? Autrement dit, quelles sont les conditions de base à respecter pour gérer ces situations d'asymétrie de projet et aboutir à des relations partenariales réussies?

\section{Conditions stratégiques de la réussite partenariale}

L'asymétrie de projet dans le partenariat Nord-Sud est donc plus que probable. Cependant, si la similitude aussi bien des facteurs de motivation que de l'engagement des partenaires est loin d'être réunie, cela ne signifie pas pour autant qu'il y a opposition absolue de projet. Nous allons effectivement montrer que, pour peu que les partenaires manifestent une réelle volonté de gérer leur accord, l'asymétrie de projet qu'il est possible d'observer peut être amplement dépassée (voir l'étude approfondie de trois cas de partenariat franco-algérien, dans l'encadré).

Selon que l'on examine la logique du partenaire du Nord ou celle de la partie du Sud, une lecture approfondie des facteurs de motivation et d'engagement fait souvent apparaître, comme nous l'avons déjà souligné, une situation d'asymétrie de projet. En ce qui concerne les trois cas étudiés, ce constat est loin d'être exceptionnel (tableau 5).

\subsection{Asymétrie de motivation et réciprocités d'intérêts}

Le premier niveau qu'il convient d'examiner dans une relation partenariale concerne les facteurs de motivations. En effet, un constat d'asymétrie de projet est simple à établir à ce niveau. L'opposition facteurs de motivation offensifs contre facteurs de motivation défensifs concerne particulièrement deux cas parmi les trois étudiés (D-A et C-L). Elle semble toutefois moins directe dans le cas E-X où les partenaires envisagent tous leur accord en vue d'atteindre certains objectifs de développement biens précis. Ce constat mérite néanmoins d'être 


\section{Cas examinés et méthodologie de l'étude}

Trois cas de partenariat entre PME algériennes et groupes industriels français sont ici retenus :

Le partenariat E-X est un accord de coopération industrielle. La relation établie repose sur un prêt de moules au partenaire algérien pour la fabrication de toute une gamme de produits petits électroménagers. Le prêt est consenti en contrepartie de l'engagement explicite de ce dernier à acheter ses composants, selon un marché de fournitures bien défini, auprès du partenaire occidental. Le début des opérations remonte à janvier 1989.

Le partenariat C-L est une entreprise conjointe d'importation, de production et de diffusion du matériel technique, conjugué à une convention d'assistance technique. Cette coentreprise a été créée en 1978 et son capital social est détenu majoritairement par le partenaire algérien $(51 \%)$.

Le partenariat D-A est un accord de cession de licence pour la fabrication de contacteurs et de relais thermiques, avec rachat d'une fraction de la production par le groupe français. Le financement du projet est imputé sur une ligne de crédits franco-algérienne. Le lancement des opérations de production est intervenu en 1990.

Pour mener à bien cette étude, nous avons opté pour une démarche en trois volets alliant analyse de la littérature et observation du terrain. Dans un premier mouvement, nous avons essentiellement favorisé le traitement de la littérature existante par l'examen aussi bien de travaux relatifs aux opérations de transferts de technologie, que des publications récentes sur les accords d'alliance entre firmes de pays développés. Dans un second mouvement, le travail engagé a également exigé le dépouillement d'un certain nombre de revues spécialisées (MOCI, Marchés tropicaux et méditerranéens, Algérie sélection, etc.) et la rencontre de responsables des milieux professionnels les plus impliqués (chambres de commerce, Proparco, CFCE, etc.).

Dans un troisième mouvement, et pour étudier de près la réalité du phénomène évoqué, nous avons choisi d'entreprendre une série d'entretiens avec plusieurs dirigeants des entreprises partenaires impliquées dans les accords retenus. Nous avons ainsi chercher à confronter les avis d'acteurs qui y sont impliqués à des degrés et à des titres différents (responsable général du projet, responsable des opérations de fabrication, assistants techniques, etc.), et ce tant chez les PME algériennes que chez les partenaires français. Un guide d'entretien, élaboré selon les aspects théoriques déjà présentés, nous a évidemment servi dans cette entreprise. Pour disposer de plus d'informations générales sur chaque cas, nous avons également entrepris un travail d'analyse de tous les documents (se rapportant aux accords en question) qui ont été mis à notre disposition par les responsables interviewés. 
Tableau 5

Facteurs d'engagement et de motivation dans le partenariat

\begin{tabular}{|c|c|c|}
\hline & Partenaires locaux & Partenaires occidentaux \\
\hline \multirow{2}{*}{$\begin{array}{l}\text { Situation } \\
\text { d'asymétrie } \\
\text { totale } \\
\text { (Partenariat C-L) }\end{array}$} & $\begin{array}{l}\text { - Pour la PME locale, le } \\
\text { partenariat est offensif. Il est } \\
\text { vécu comme une action pilote } \\
\text { pour acquérir certains savoirs } \\
\text { et savoir-faire cruciaux, en vue } \\
\text { d'intégrer localement la } \\
\text { fabrication de nouvelles } \\
\text { gammes de produits. }\end{array}$ & $\begin{array}{l}\text { - Pour contourner l'inter- } \\
\text { diction d'importation de } \\
\text { certains équipements par les } \\
\text { firmes étrangères, le groupe } \\
\text { français a choisi de s'associer } \\
\text { avec une entreprise locale } \\
\text { pour continuer d'approvi- } \\
\text { sionner le marché algérien. }\end{array}$ \\
\hline & $\begin{array}{l}\text { - L'engagement est actif. Les } \\
\text { contributions apportées sont } \\
\text { diverses et spécifiques } \\
\text { (programmes de formation } \\
\text { spécifique, équipements } \\
\text { spécialisés, etc). }\end{array}$ & $\begin{array}{l}\text { - L'engagement est passif. } \\
\text { L'apport en capital est peu } \\
\text { significatif et l'image de } \\
\text { marque du groupe n'est en } \\
\text { aucun cas affectée. Même la } \\
\text { lecture des documents } \\
\text { diffusés par la coentreprise } \\
\text { ne permet pas l'identification } \\
\text { du coassocié occidental. }\end{array}$ \\
\hline
\end{tabular}

\begin{tabular}{|c|c|c|}
\hline \multirow{2}{*}{$\begin{array}{l}\text { Situation } \\
\text { d'asymétrie } \\
\text { d'engagement } \\
\text { (Partenariat E-X) }\end{array}$} & \multicolumn{2}{|c|}{$\begin{array}{l}\text { Le partenariat est ici offensif pour les deux parties. Le partenaire } \\
\text { local vise l'acquisition de certains savoir-faire cruciaux en vue de } \\
\text { lancer sa propre gamme de petits électroménagers. Les facteurs de } \\
\text { motivations des groupes industriels français semblent en revanche } \\
\text { très liés à la volonté de s'assurer annuellement un volume d'affaires } \\
\text { non négligeable (ventes de composants). }\end{array}$} \\
\hline & $\begin{array}{l}\text { L'implication est significative } \\
\text { (formation spécialisée, } \\
\text { programmes d'assistance } \\
\text { technique, etc.) }\end{array}$ & $\begin{array}{l}\text { L'engagement est sans risques } \\
\text { majeurs. Il implique une simple } \\
\text { mise à disposition de moules qui } \\
\text { sont, par ailleurs, démodés et } \\
\text { totalement amortis. }\end{array}$ \\
\hline \multirow{2}{*}{$\begin{array}{l}\text { Situation } \\
\text { d'asymétrie } \\
\text { de facteurs } \\
\text { de motivation } \\
\text { (Partenariat } \mathrm{C}-\mathrm{L} \text { ) }\end{array}$} & $\begin{array}{l}\text { La firme locale dispose d'un } \\
\text { parc machines important, mais } \\
\text { qui reste imparfaitement } \\
\text { exploité. Son partenariat ici } \\
\text { examiné est vécu comme une } \\
\text { action réactive en vue de } \\
\text { rationaliser le fonctionnement et } \\
\text { d'atteindre un niveau } \\
\text { d'utilisation satisfaisant des } \\
\text { capacités productives. }\end{array}$ & $\begin{array}{l}\text { Le partenariat présente une } \\
\text { nature offensive. Pour les } \\
\text { responsables interviewés, } \\
\text { ce projet reflète une conduite } \\
\text { offensive en vue de pénétrer } \\
\text { le marché algérien, soit par vente } \\
\text { de composants ou d'équipements, } \\
\text { soit par signature de conventions } \\
\text { d'assistance technique. }\end{array}$ \\
\hline & \multicolumn{2}{|c|}{$\begin{array}{l}\text { L'attitude des partenaires est active. En dehors des équipements } \\
\text { spécifiques acquis, la PME algérienne s'est, par exemple, engagée dans } \\
\text { un profond processus de réorganisation de son activité productive. } \\
\text { Le partenaire français apporte, de son côté, sa notoriété et l'engage- } \\
\text { ment de rachat d'une fraction de la production. }\end{array}$} \\
\hline
\end{tabular}


nuancé, eu égard aux réciprocités d'intérêts ${ }^{4}$ qu'il est possible de détecter dans ces conduites concertées.

La similitude de projet dans le partenariat E-X, par exemple, n'est qu'apparente. L'intérêt de base du partenaire français réside dans la vente de sous-ensembles de produits. Le partenaire local affiche, pour sa part, le souhait de maîtriser le processus de production et de dépasser la simple exécution d'activités de montage. En réalité, la divergence est donc loin d'être absente. Le groupe français est en effet peu enthousiaste à l'idée que son partenaire maîtrise rapidement les processus de production, pour se situer sur de réels processus de fabrication, car les ventes de composants rapportent davantage que les livraisons de simples fournitures de base. L'interdépendance d'intérêts dans cet accord est, en somme, réduite aux seuls éléments commerciaux : vente de composants pour les locaux contre vente de produits assemblés pour les occidentaux.

Dans le partenariat D-A, l'asymétrie de projet est certes très marquée (logique défensive pour l'un et logique offensive pour l'autre), bien qu'elle n'exclue pas la présence de réciprocités d'intérêts. L'accord «d'achat en retour » (buy-back) convenu indique effectivement que la partie étrangère est obligée de racheter à la PME locale une fraction de la production, équivalente à $32 \%$ de la valeur totale des achats de composants effectués par cette dernière. La firme algérienne est ainsi à la fois cliente du partenaire français pour l'achat de composants (ou de technologies) et sous-traitante pour la fabrication d'un contingent donné. Aussi, afin de s'assurer, d'une part, que la fraction de la production rachetée soit d'une qualité supérieure et, d'autre part, que le volume de vente de composants soit important, le partenaire occidental a tout intérêt à ce que son partenaire local progresse rapidement dans son processus d'apprentissage technologique. Par cette formule, les partenaires ont donc su faire converger leurs visions. La réciprocité d'intérêts définie est, de surcroît, plus globale que dans le cas E-X ; elle se révèle non seulement associée aux possibilités d'atteindre certains objectifs commerciaux et financiers, mais aussi à celles d'acquérir certains savoirs et savoir-faire.

Soulignons que, dans le partenariat Nord-Sud, la divergence des facteurs de motivations (ou leur convergence), finalement importe peu ; ce qui détermine la nature des réciprocités d'intérêts possibles dans ce type de conduite, ce sont plutôt les conditions de base de l'accord. Selon les termes contractuels

4. Au-delà des intérêts communs qui peuvent être distingués (obtention d'un chiffre d'affaires donné, partage des risques), l'entrecroisement d'intérêts dont il est ici question suppose que chaque partie, pour atteindre ses propres objectifs, doit être motivée et même contrainte d'œuvrer pour aider son partenaire à atteindre les siens. 
de la relation, les réciprocités d'intérêts de départ peuvent être élargies ou réduites aux seules préoccupations commerciales. Dans le partenariat C-L, par exemple, la formule adoptée pour calculer la rétribution annuelle du partenaire étranger au titre de son assistance technique a fini par transformer l'enjeu de cette association. La rémunération pratiquée (modulable dans sa partie variable en fonction du chiffre d'affaires obtenu et d'un pourcentage appliqué au montant total des salaires bruts versés au personnel expatrié) n'autorise, en fait, qu'une seule interdépendance positive : le volume d'activité réalisé. L'apprentissage n'est en revanche nullement recherché. La partie étrangère est effectivement peu intéressée à s'investir dans cette direction, car cela risque d'entraîner le remplacement rapide de son personnel expatrié. Son intérêt est plutôt dans le maintien d'un grand nombre de détachés afin de s'assurer une base de calcul toujours importante.

Les partenaires doivent, par conséquent, réfléchir dès l'instant de l'établissement de leur relation sur les mécanismes à introduire au sein de leur accord pour garantir l'interdépendance totale d'intérêts ; les formules imaginables à ce propos sont très nombreuses. L'un des mécanismes possibles est d'indexer partiellement la rétribution du partenaire occidental (au titre de son assistance technique par exemple) à l'atteinte d'une suite d'objectifs : chiffre d'affaires, maîtrise technologique satisfaisante, lancement de nouveaux produits, etc. Une deuxième formule consiste à conditionner le renouvellement du contrat d'assistance technique ou d'achat de composants et fournitures à l'accomplissement d'un premier niveau de maîtrise des opérations. Une troisième solution, d'un niveau supérieur, réside dans le choix d'accords de partenariat impliquant une réciprocité de fait des rôles. L'exemple à indiquer, à ce propos, est le cas d'accords de partenariat avec rachat d'une fraction de la production, où chaque partenaire est appelé à intervenir dans un rôle en rapport direct avec la tâche de l'autre partie.

En somme, la première règle à respecter pour gérer l'asymétrie de projet qui caractérise les relations partenariales Nord-Sud est de s'assurer que tous les partenaires manifestent ensemble une série d'utilités réciproques. Le codéveloppement de ces derniers ne peut être garanti qu'en présence d'une telle condition de départ. Cependant, si des intérêts réciproques peuvent être établis et définis à l'origine de la relation (au moment de la signature de l'accord), il est logique de penser que ceux-ci doivent être sans cesse réexaminés, car ils ne sont jamais définitivement acquis.

\subsection{Réciprocités d'intérêts et dynamique de l'accord}

S'il est vrai que le partenariat, par définition, élimine les difficultés ne résultant pas d'un antagonisme réel des intérêts des partenaires, il ne reste pas moins que 
sa réalité à long terme pourrait être source de conflits ${ }^{5}$. À long terme, toute mutation dans l'environnement partenarial peut devenir source de différends, dans la mesure où elle risque d'entraîner des modifications dans la position des parties à l'égard de l'objet de l'accord (Harrigan, 1988).

L'objectif ici est d'analyser, dans une perspective dynamique, l'évolution stratégique des relations partenariales étudiées. Nous nous efforcerons de comprendre dans quelle mesure les utilités réciproques de départ ont été élargies après l'apparition des principaux différends (tableau 6).

TABleau 6

Évolutions stratégiques intervenues dans les accords traités

\begin{tabular}{|c|c|c|}
\hline Cas & Différends stratégiques survenus & Changements majeurs apportés \\
\hline $\begin{array}{c}\text { Partenariat } \\
\text { D-A }\end{array}$ & $\begin{array}{l}\text { - Pour des raisons d'évolution de } \\
\text { gammes, le produit accepté par la } \\
\text { partie étrangère pour faire l'objet } \\
\text { de rachat d'une fraction de la } \\
\text { production s'est révélé rapidement } \\
\text { non adapté aux besoins de cette } \\
\text { dernière. }\end{array}$ & $\begin{array}{l}\text { - Élargissement de la coopération } \\
\text { vers des produits plus complexes. } \\
\text { - Modification de l'accord de } \\
\text { compensation et évolution du rachat } \\
\text { vers une partie de la production sur } \\
\text { laquelle les partenaires sont dans de } \\
\text { meilleures dispositions pour obtenir } \\
\text { de bonnes marges. }\end{array}$ \\
\hline $\begin{array}{l}\text { Partenariat } \\
\text { E-X }\end{array}$ & $\begin{array}{l}\text { - Volonté de la partie locale de faire } \\
\text { diversifier rapidement sa gamme de } \\
\text { produits fabriqués, tout en portant } \\
\text { la coopération vers les mêmes } \\
\text { produits que ceux commercialisés } \\
\text { par le partenaire sur ses propres } \\
\text { marchés. }\end{array}$ & $\begin{array}{l}\text { - Renouveau dans la coopération } \\
\text { pour permettre au partenaire } \\
\text { algérien de varier sa gamme de } \\
\text { production. } \\
\text { - Toutefois, les produits fabriqués } \\
\text { restent encore limités à ceux arrivés } \\
\text { au stade de maturation sur le } \\
\text { marché international. }\end{array}$ \\
\hline & $\begin{array}{l}\text { - Désaccord de la partie étrangère } \\
\text { quant à la politique menée } \\
\text { en matière de distribution } \\
\text { de dividendes. }\end{array}$ & $\begin{array}{l}\text { - Manque d'implication du partenaire } \\
\text { étranger au fur et à mesure que } \\
\text { ses ventes d'équipements à } \\
\text { l'entreprise mixte diminuaient. }\end{array}$ \\
\hline $\begin{array}{c}\text { Partenariat } \\
\text { C-L }\end{array}$ & $\begin{array}{l}\text { - Divergence de visions quant aux } \\
\text { programmes à poursuivre dans } \\
\text { l'optique d'intégrer de plus en plus } \\
\text { la fabrication de nouvelles gammes } \\
\text { de produits. }\end{array}$ & $\begin{array}{l}\text { - Appel à d'autres partenaires } \\
\text { étrangers pour lancer quelques } \\
\text { programmes de fabrication, qui } \\
\text { restent toutefois de minimes } \\
\text { opérations de montage de kits } \\
\text { importés. }\end{array}$ \\
\hline
\end{tabular}

5. Certains auteurs soulignent que l'importance des conflits est fonction de la culture de l'entreprise locale et de la position de leader ou non du partenaire occidental. Les conflits sont faibles, en particulier, dans le cas d'associations entre partenaires du Sud de culture commerçante, qui ne se soucient guère de mettre en œuvre des politiques d'apprentissages technologiques, et partenaires du Nord, en position marginale ou secondaire sur le marché. Ils sont, en revanche, très probables quand la relation réunit des entreprises de culture industrielle manifestant une volonté de maitrise technologique et des partenaires occupant une place de leader sur le marché mondial. 
Le partenariat ne se déroule donc pas sans obstacles ni émergence de différends. La dynamique du partenariat fait effectivement évoluer les orientations, et les solutions apportées peuvent être de diverses natures.

Dans le partenariat D-A, le différend stratégique majeur survenu est lié à la difficulté de déterminer quel sera le produit objet de la compensation (rachat d'une fraction de la production). Pour des raisons d'évolution de gammes, le produit défini au départ s'est révélé rapidement non conforme aux besoins de la partie étrangère. De surcroît, la partie locale ne peut accepter l'annulation de ce rachat qui, en fait, lui apporte une solution partielle à ses problèmes de financements en devises d'importations de composants et de fournitures. Aussi, pour sortir de cette impasse, les parties impliquées ont décidé, après de laborieuses négociations, de faire évoluer ce rachat vers une partie de la production sur laquelle elles sont dans de meilleures dispositions pour obtenir de bonnes marges. De cette manière, les partenaires sont également parvenus à élargir l'objet de leur coopération vers des produits plus complexes.

De nouveaux intérêts réciproques ont été, par conséquent, définis, permettant ainsi à ce partenariat d'évoluer d'un premier niveau de coopération à un second mutuellement plus avantageux. En effet, le volume annuel de vente de composants que peut désormais réaliser le partenaire français est de plus en plus conséquent. Les responsables locaux soulignent, de leur côté, qu'avec l'élargissement de la coopération vers des produits plus complexes, la rationalisation de l'outil de production est plus significative. Le projet s'est effectivement concrétisé moyennant l'acquisition de certains équipements spécifiques et l'utilisation rationnelle des capacités déjà installées chez la PME locale. Notons que cette dernière n'a pas cherché à acquérir tous les équipements nécessaires ; elle s'est plutôt efforcée de trouver des possibilités d'usage du parc machine disponible. Le site de production utilisé se trouve, en outre, au sein même des bâtiments industriels existants, et la totalité de l'effectif affecté au projet provient d'une sélection rigoureuse du personnel présent dans l'entreprise. Cela reflète, de façon globale, une attitude de coopération élargie basée sur la volonté de faire évoluer l'accord vers de nouveaux espaces coopératifs dans le respect des solidarités réciproques.

Dans le partenariat E-X, le conflit a trait à la définition de la gamme de produits fabriqués. Il est apparu lorsque la PME algérienne a manifesté le désir de diversifier sa gamme de produits et de porter la coopération vers des productions de «dernier cri »; c'est sur ce dernier aspect que le désaccord est survenu. Le partenaire local doit, selon les responsables français interviewés, se concentrer sur la maîtrise des technologies déjà mises en place; d'autres étapes préalables et nécessaires doivent être franchies avant qu'il puisse envisager la fabrication de produits plus complexes. Pour résoudre ce différend, les 
partenaires ont fini par adopter une solution médiane. Ils ont décidé d'élargir leur coopération vers de nouvelles productions, tout en continuant de limiter la gamme de produits fabriqués à ceux arrivés au stade de maturation sur les marchés internationaux. Ce compromis permet, certes, aux parties de régler leur différend, mais n'apporte pas une modification majeure à l'accord d'origine. Le partenaire local est toujours tenté de renforcer son apprentissage dans le domaine de la production afin d'intégrer rapidement la fabrication de nouveaux produits à la mode, alors que la partie française s'efforce de vendre davantage de composants tout en cherchant à éviter toute éventualité de concurrence en retour.

Dans le partenariat C-L, la coopération étroite observée durant les premières années de la coentreprise, à travers notamment la convention d'assistance technique conclue, n'a duré qu'une période restreinte. Une fois que le personnel expatrié a été presque totalement remplacé par des nationaux, les occasions d'interaction entre les deux partenaires ont nettement diminué. En outre, cette situation s'est nettement dégradée après la décision prise par le partenaire algérien, après l'instauration d'une série de mesures gouvernementales, de diversifier ses fournisseurs de matériels techniques (recours à des fournisseurs offrant des produits peut-être de qualité moyenne, mais à des prix compétitifs). Les projets d'intégration de nouvelles gammes de produits ont été, par ailleurs, retardés devant le désintéressement et le peu d'enthousiasme manifestés par le groupe français ${ }^{6}$. La coentreprise s'est vue alors contrainte de faire appel à d'autres partenaires (italiens et suisses) et de se contenter de lancer de simples activités de montages de kits importés chez les partenaires en question. Avec le partenaire français, la relation s'est en fin de compte transformée en une simple occasion d'échanges commerciaux sporadiques. Elle est cependant maintenue, car la PME algérienne trouve toujours intérêt à faire appel à l'assistance technique de son partenaire (notamment dans le domaine médical), alors que ce dernier considère qu'il est globalement plus utile d'avoir une entrée, même passive, que de perdre toute occasion de présence en Algérie.

Tout bien considéré, nous convenons avec Mappa (1991), que rien d'essentiel ne se fait dans l'angélisme, mais encore faut-il pouvoir gérer les relations conflictuelles. L'apparition de conflits dans les partenariats ne signifie pas qu'ils ont échoué : la réussite d'une opération de partenariat ne se juge pas au fait que les partenaires ne connaissent pas de différends (Ingham, 1990). Tout dépend en réalité de l'attitude qui est privilégiée pour les résoudre, surtout lorsqu'ils se manifestent avec force.

6. Cette passivité, soulignent les responsables français rencontrés, est également liée à l'obstination du partenaire majoritaire de ne pas accepter la distribution de dividendes. 
À l'image des cas examinés, il est possible de distinguer trois conduites partenariales majeures ${ }^{7}$ dans les opérations conjointes (tableau 7).

TABLEAU 7

Comportements stratégiques relevés

\begin{tabular}{|c|c|c|c|}
\hline & $\begin{array}{l}\text { Coopération } \\
\text { élargie }\end{array}$ & $\begin{array}{l}\text { Attitude de } \\
\text { compromis }\end{array}$ & $\begin{array}{l}\text { Attitude de } \\
\text { négligence }\end{array}$ \\
\hline $\begin{array}{l}\text { Caractéristiques } \\
\text { principales }\end{array}$ & $\begin{array}{l}\text { Recherche continue } \\
\text { de nouveaux et } \\
\text { larges espaces } \\
\text { coopératifs. }\end{array}$ & $\begin{array}{l}\text { Dénouement des } \\
\text { différends sans pour } \\
\text { autant permettre } \\
\text { l'augmentation de } \\
\text { l'efficacité globale } \\
\text { de l'accord. }\end{array}$ & $\begin{array}{l}\text { Résignation dans un } \\
\text { rôle passif et refus } \\
\text { d'engager le moindre } \\
\text { investissement } \\
\text { supplémentaire. }\end{array}$ \\
\hline $\begin{array}{l}\text { Réciprocités } \\
\text { d'intérêts établies }\end{array}$ & $\begin{array}{l}\text { Élargissement des } \\
\text { réciprocités de départ }\end{array}$ & $\begin{array}{l}\text { Simple déplacement des } \\
\text { réciprocités d'origine }\end{array}$ & $\begin{array}{l}\text { Appauvrissement des } \\
\text { réciprocités de départ }\end{array}$ \\
\hline Cas concernés & D-A & E-X & $C-L$ \\
\hline
\end{tabular}

Dans toutes ces situations, il y a certes juxtaposition d'éléments coopératifs et d'éléments opportunistes. Toutefois, le dosage observé est manifestement inégal. Dans certaines conduites, ce sont les éléments coopératifs qui prédominent alors que dans d'autres, ce sont plutôt les aspects opportunistes qui se manifestent avec force. Aussi, selon le style de conduite favorisé, le devenir du rapprochement partenarial risque d'être fort différent.

Les conduites de coopération sous-tendent généralement des comportements très constructifs. Les interdépendances d'objectifs sont perçues comme importantes et nécessaires afin de propulser le partenariat vers plus d'échanges féconds. Les différends sont résolus de manière à ce que chaque partie arrive à la satisfaction de ses intérêts, tout en manifestant le désir de permettre à l'autre d'obtenir les siens. Ainsi, dans le partenariat D-A, la décision des parties de faire évoluer l'objet de la compensation vers un produit plus complexe permet l'augmentation de l'efficacité globale de l'accord par l'élargissement des réciprocités d'intérêts. Le nouveau produit désigné offre effectivement de meilleures marges et situe désormais l'interdépendance d'intérêts en rapport avec des aspects de savoirs et de savoir-faire plus complexes.

Le comportement de compromis se distingue par une certaine équivalence entre les facteurs coopératifs et les facteurs opportunistes. Les solutions

7. Cette distinction repose sur le modèle des styles de comportements dans le règlement des conflits interpersonnels développé par Thomas (1975), qui soutient que ceux-ci évoluent, en général, en fonction de l'attitude de chacune des parties engagées sur deux dimensions : la considération pour soi et la considération pour l'autre. 
favorisées restent cependant distributives ; la relation est perçue comme un jeu à somme fixe. Dans le cas E-X, le compromis négocié pour régler le différend survenu n'apporte qu'une modification mineure à l'accord d'origine. Les termes de la coopération sont sans modification réelle (l'accord implique toujours la même gamme de produits) et les réciprocités d'intérêts établies restent toujours les mêmes : de simples interdépendances commerciales. Le compromis n'engendre pas l'établissement de nouvelles et larges réciprocités d'intérêts. Il permet seulement le déplacement des réciprocités de départ. Il n'en demeure pas moins une conduite critique quand, à défaut d'en établir de nouvelles, il est important de maintenir celles déjà établies.

Les conduites de négligence renvoient à des comportements dépourvus de coopération. Les interdépendances d'objectifs sont jugées globalement insignifiantes, et l'enjeu de la relation est perçu comme peu important. Il est clair que ce type de conduites ne peut procurer un enrichissement mutuel des partenaires. La passivité du partenaire français dans le cas C-L se manifestant dans son refus de participer à l'effort de la coentreprise algérienne de fabriquer localement certains produits est à ce propos très significative. Cette conduite a fini, en effet, par appauvrir les interdépendances d'objectifs de base, pour ensuite transformer l'association en une simple suite d'échanges commerciaux sporadiques. Il ne peut y avoir de réciprocités d'intérêts durables dès que l'un des partenaires cesse d'exercer son rôle et refuse de s'engager réellement dans le processus coopératif.

En définitive, la dynamique du partenariat ne doit pas être vue comme une contrainte, mais plutôt comme un moyen pour les partenaires de renforcer leurs relations et d'envisager de nouvelles réciprocités d'intérêts. En conséquence, c'est par la volonté des partenaires à faire évoluer de manière durable leur relation vers la détermination de nouvelles combinaisons d'activités avantageux pour tous qu'ils peuvent assurer un succès global à leur partenariat. En effet, la réussite globale exige la définition d'intérêts réciproques divers impliquant aussi bien des aspects commerciaux que des éléments techniques et managériaux. À ce propos, le cas D-A, reflétant une conduite de coopération élargie, est très significatif. Les résultats globaux obtenus (fin 1994) après plusieurs années d'accord sont évalués comme très satisfaisants. Par ailleurs, et à l'image des deux autres cas, il est logique de penser qu'une simple réciprocité d'intérêts commerciaux ne peut, à elle seule, garantir, au mieux, qu'un simple partage d'opportunités de marchés (tableau 8).

La question que l'on doit se poser à présent concerne les conditions qui peuvent favoriser l'apparition de tel ou tel comportement. À ce propos, il est intéressant de revenir à l'implication stratégique des partenaires dans ces conduites. 
TABLEAU 8

Réciprocités d'intérêts établies et succès partenarial obtenu

\begin{tabular}{|c|c|c|}
\hline Cas concernés & $\begin{array}{c}\text { Réciprocités d'intérêts } \\
\text { établies }\end{array}$ & Niveau de succès obtenu \\
\hline $\begin{array}{l}\text { - Partenariat C-L } \\
\text { - Partenariat E-X }\end{array}$ & $\begin{array}{l}\text { Simples réciprocités } \\
\text { d'intérêts commerciaux. }\end{array}$ & $\begin{array}{l}\text { Le succès est commercial : simple } \\
\text { partage d'opportunités de marchés. } \\
\text { Le C.A. est en croissance importante, } \\
\text { alors que la maîtrise technique est } \\
\text { limitée à des opérations de montages } \\
\text { de kits importés. La qualité des produits } \\
\text { fabriqués reste en outre peu } \\
\text { satisfaisante. }\end{array}$ \\
\hline - Partenariat D-A & $\begin{array}{l}\text { Réciprocités } \\
\text { d'intérêts plus larges } \\
\text { impliquant aussi } \\
\text { bien des aspects } \\
\text { commerciaux } \\
\text { que des éléments } \\
\text { techniques } \\
\text { et organisationnels. }\end{array}$ & $\begin{array}{l}\text { Le succès à ce niveau est global: } \\
\text { l'exploitation des systèmes technolo- } \\
\text { giques acquis est largement satisfaisante, } \\
\text { le respect des normes de qualités les plus } \\
\text { strictes est garanti, la maintenance des } \\
\text { équipements est entièrement assurée par } \\
\text { le personnel autochtone. L'interaction } \\
\text { avec le partenaire s'est, en outre, soldée } \\
\text { par la mise en exploitation de nouvelles } \\
\text { activités. On note également une } \\
\text { augmentation sensible du volume } \\
\text { d'affaires réalisé. }\end{array}$ \\
\hline
\end{tabular}

\subsection{Asymétrie de l'engagement et conduite partenariale}

Pour le bon déroulement du partenariat, il est raisonnable de penser que tout partenaire doit être impliqué de manière à ce que l'échec du projet risque d'entraîner, pour chacun, la perte de toute une série d'apports ${ }^{8}$. En présence d'un risque de perte conséquent, les partenaires seraient plus tentés de maintenir des liaisons positives que de favoriser des pratiques opportunistes pouvant accélérer l'échec des rapports instaurés. Cela est d'autant plus vrai que l'engagement bilatéral des partenaires est crédible et passe par des contributions spécifiques dont la valeur future en cas de réinvestissement est moindre (Williamson, 1985).

Dans les cas étudiés ici, cet aspect des choses est évidemment bien admis. Chaque partie prenante est impliquée de telle manière que l'échec du projet

8. Rappelons que par apports, nous entendons toutes les contributions et prestations directes ou indirectes fournies pour mettre en œuvre le projet de partenariat et assurer son fonctionnement normal. 
risque de lui faire perdre toute une série de contributions ${ }^{9}$. Cependant, un retour sur le tableau 5 et un examen encore plus approfondi des contributions des partenaires peuvent nous renseigner davantage sur le déroulement de ces cas.

Dans le partenariat D-A, l'engagement du partenaire français de racheter une fraction de la production est une contribution qui peut lui faire courir plusieurs risques. Effectivement, l'allié occidental est loin d'être à l'abri d'un malentendu ou d'une indélicatesse de son partenaire. Les produits, objets de la compensation, peuvent être non conformes aux échantillons, non adaptés aux besoins ou incompatibles avec les normes de qualité exigées. Cela est d'autant plus grave que ces produits sont appelés à être intégrés dans les productions du groupe (ou de ses filiales). De façon indirecte, l'accord peut également affecter le partenaire étranger dans son image et sa notoriété, puisque la marque de ce dernier figure sur tous les produits issus de ce partenariat. Par conséquent, une mauvaise production et une moindre fiabilité peuvent mettre à mal sa réputation et sa crédibilité, si ce n'est pas tous ses marchés, du moins sur le marché algérien. Ce discrédit peut de surcroît avoir des conséquences néfastes, si l'on sait que ce groupe est présent à l'échelle des pays de la région dans de nombreux domaines d'activités.

Pour la PME algérienne, au-delà des équipements spécifiques acquis et des programmes de formation spécialisée engagés, l'implication s'est également traduite par une réorganisation profonde de la production pour opérer des regroupements technologiques. La règle respectée consiste à trouver une meilleure complémentarité entre le parc machines disponible et les équipements spécifiques nouvellement acquis, et à s'assurer d'un meilleur apprentissage en concentrant l'exploitation de chaque catégorie de techniques dans un lieu unique. L'activité de partenariat ainsi établie est désormais en interaction totale avec le reste des activités de l'entreprise. En conséquence, l'exécution de cet accord peut affecter directement ou indirectement l'efficacité globale du système d'offre de cette PME.

L'implication des partenaires est donc à effets rétroactifs continus, en ce sens qu'ils risquent tous les deux de subir, en retour, des conséquences négatives larges en cas d'accrocs. Plus que la simple mise en apport d'une série d'actifs même spécifiques (implication active), on note ici une symétrie

9. Certaines ressources réunies sont, de surcroît, sans grande valeur dans d'autres situations. C'est le cas, par exemple, des contributions en termes de connaissances du marché algérien et d'introductions auprès des autorités locales, des équipements spécialisés acquis et qui ne peuvent être mis en usage qu'en rapport avec le parc machines déjà disponible, des sommes déboursées afin d'assurer une formation spécialisée au personnel autochtone et les programmes de formation engagés en vue de préparer les expatriés aux particularités du milieu d'accueil. 
d'engagement qui se reflète dans des contributions particulières visant directement ou indirectement les activités propres des parties. Aussi, il est vraisemblable que le comportement de coopération élargie observé, dans ce cas, soit lié à cette situation particulière d'engagement. Afin de permettre une meilleure issue à leur partenariat et d'éviter ainsi les conséquences d'un mauvais fonctionnement de celui-ci, les partenaires ont préféré chercher l'élargissement de la collaboration. Le préjudice en cas d'échec dans ces conditions est nettement plus dommageable et dépasse la seule perte des actifs investis dans l'accord.

À l'image de ce cas, il semble donc que le comportement de coopération élargie ne peut être produit qu'en présence de cette symétrie particulière d'engagement. L'absence d'une telle symétrie peut, en revanche, pousser les partenaires à tenter de récupérer aussi rapidement que possible leur mise de départ. L'attitude sera alors coopérante durant les premières années de l'accord et finira par disparaître dès que l'un des partenaires aura pu faire fructifier son engagement d'origine ${ }^{10}$. Cela est crucial pour comprendre les attitudes de négligence et de compromis observées dans les deux autres cas.

Dans le partenariat C-L, les produits fabriqués portent la marque de la coentreprise créée. Il semble également impossible, à la lecture de tout document diffusé par cette dernière, de l'identifier comme une entreprise mixte et de relever l'identité du coassocié étranger: elle est simplement présentée comme une filiale du partenaire local. L'engagement du groupe français est donc restreint au capital de départ investi et n'implique pas rétroactivement le fonctionnement de ses propres activités ou de son image de marque. La conduite de négligence auparavant évoquée dans ce cas peut être expliquée par l'asymétrie d'engagement ainsi constaté. N'ayant pas à craindre de subir des effets rétroactifs négatifs en cas de mauvais fonctionnement futur de la coentreprise, le partenaire occidental s'est rapidement réfugié dans un rôle très passif (devenant un sleeping partner), notamment après qu'il soit parvenu à rentabiliser largement sa mise de départ. Il recevait effectivement une

10. Ce risque est d'autant plus réel que, dans le partenariat Nord-Sud, il y a de fortes probabilités que les deux parties n'aient pas exactement les mêmes gains selon qu'on se situe au début de l'opération ou après quelques années d'exercice (distribution inégale des bénéfices). L'essentiel des gains au début de l'opération est généralement empoché par le partenaire du pays développé. Cette situation tend ensuite à s'équilibrer, voire à s'inverser en faveur de la partie du Sud, une fois que celle-ci est en mesure de faire des apprentissages et de maîtriser les différents aspects de l'opération. À ce propos, certains auteurs font remarquer qu'il est utile de s'entendre dès le départ sur un système de balancier: un avantage relatif pour l'un des partenaires dans une première phase de l'accord doit être compensé par un avantage relatif pour l'autre dans une seconde étape (Urban et Vendemini, 1994, p. 17). 
rétribution annuelle conséquente en vertu de son assistance technique et s'assurait, à lui seul, durant plusieurs années, $85 \%$ des importations de la coentreprise locale. En somme, il s'avère que ce partenariat n'est en réalité qu'une simple relation commerciale déguisée destinée à créer un flux régulier d'échanges sous la forme de services et d'équipements vendus à l'entreprise conjointe.

Le même constat est valable en ce qui concerne le partenariat E-X. Pour la partie occidentale, l'opération reste sans risque significatif puisque limitée à la détérioration des jeux de moules prêtés, dont elle ne se sert plus pour ses propres activités et qui sont d'ailleurs anciens et totalement amortis. Les produits fabriqués portent, par ailleurs, la marque de la PME algérienne et, en aucune manière, il n'est fait référence au partenaire français. Il semble ainsi que c'est cette asymétrie d'engagement qui a freiné les parties dans leur processus de coopération. Les effets en retour liés à l'engagement de la partie française, si l'accord tourne mal, ne peuvent viser que de simples pertes de contrats de fournitures. Aussi, la coopération que prône cette dernière est réduite à la volonté de sauvegarder un volume conséquent de ventes de composants. La conduite de compromis constatée n'est ainsi favorisée que pour respecter cette volonté (élargissement de la gamme, mais en produisant toujours le même type de produits).

En définitive, il convient d'indiquer qu'un partenariat fondé sur un engagement rétroactif risqué est de nature à favoriser des comportements de coopération élargie. Plus l'engagement des parties est symétrique et se reflète dans des éléments en liaison directe avec leurs activités propres, plus ces derniers seront motivés à faire évoluer leur coopération. Il en découle alors une certaine stabilité de la relation, de telle sorte que les comportements de non-coopération deviennent sensiblement peu probables. Le risque que l'un des partenaires se retranche dans un comportement de négligence en refusant d'engager le moindre effort supplémentaire, parce qu'il estime que ses objectifs sont déjà atteints, devient également très réduit. En effet, avec un tel engagement, l'intérêt de coopérer n'est pas uniquement lié à la réalisation de ses propres objectifs, mais aussi à l' aboutissement efficace de l'accord afin d'éviter les éventuels dysfonctionnements rétroactifs nuisibles.

Cependant, en dépit du degré de l'engagement et de son caractère rétroactif risqué en cas de mauvais fonctionnement, les conduites coopératives dans le partenariat Nord-Sud sont également liées à plusieurs autres facteurs. Le dépassement des asymétries de projet par l'élargissement de la coopération et la définition de nouvelles et larges réciprocités d'intérêts peut également dépendre, par exemple, de la spécificité et de l'irréversibilité des apprentissages organisationnels déjà accomplis, du degré de confiance déjà instauré et de l'importance du futur de l'accord (Wolfe, 1995). 
Tout bien considéré, la présente contribution ne propose qu'une ébauche d'un nombre de recommandations à respecter pour que le partenariat interentreprises Nord-Sud suscite autant d'occasions de coopérations que de partages positifs de larges gains. Une investigation plus poussée, menée sur la base d'un échantillon plus représentatif, est évidemment nécessaire pour renforcer la validité de l'analyse.

\section{Conclusion}

Le partenariat interentreprises Nord-Sud se reflète souvent dans un projet asymétrique dans la mesure où les objectifs visés par les deux blocs de partenaire sont rarement similaires. Aussi, pour éviter sa transformation en une relation opportuniste servant les intérêts de l'une des parties au détriment de l'autre, il est primordial que les objectifs fixés à l'origine de l'accord soient interdépendants. Plus il y a «entrelacement d'intérêts », plus le comportement coopératif est probable. La différence de motivations et d'objectifs n'est pas en soi un obstacle, pourvu qu'il soit possible de créer une communauté d'intérêts basée sur la réciprocité d'intérêts. Par conséquent, il est de la plus grande importance, pour les protagonistes en relation, de s'efforcer d'instaurer au sein de leur accord des mécanismes (formules de rétribution, par exemple) induisant la réciprocité d'intérêts recherchés. De surcroît, pour arriver à une réussite partenariale globale (pas seulement commerciale), la réciprocité en question doit concerner aussi bien des aspects commerciaux que des éléments technologiques et managériaux.

Le partenariat est évolutif. La dynamique de l'accord engendre souvent des modifications dans la situation et le pouvoir réciproque des parties et pourrait alors constituer une source d'instabilité. Il en découle la nécessité pour celles-ci de chercher à continuellement élargir leur collaboration vers de nouveaux espaces coopératifs, afin de renforcer les interdépendances d'objectifs de départ ou d'en instaurer d'autres. Cependant, ces conduites coopératives dans le partenariat sont loin d'être systématiques. Leur présence renforcée semble être associée, en partie, à un engagement actif et conséquent dont les effets en retour, en cas de mauvais fonctionnement de l'accord, risquent d'affecter négativement les activités propres des partenaires.

\section{Bibliographie}

APRODI (1989), «La Coopération industrielle Internationale : l'Expérience APRODI », Association pour la promotion du développement industriel, Paris.

CNUCED (1984), « Modalités des transferts de technologie effectués au profit de pays en développement par des petites et moyennes entreprises : monographie sur les coentreprises avec participation au capital social et les accords de technologie en Amérique latine », New York, Nations Unies. 
CNUCED (1990), «Les faits nouveaux intervenus récemment dans le domaine de la technologie et leurs rapports avec les négociations relatives au projet de Code international de conduite pour le transfert de technologie », Étude du secrétariat de la CNUCED, TD/CODE TOT/55, octobre.

CONTRACTOR, F.J. et P. LORANGE (1988), « Competition vs. cooperation : a benefit / cost framework for choosing between fully-owned investments and cooperative relationships », Management International Review, Édition spéciale, p. 5-18.

DARREON, J. et S. FAIÇAL (1993), «Les enjeux des partenariats stratégiques entre grandes entreprises et PME », Revue Française de Gestion, n ${ }^{\circ}$ 95, p. 104-115.

Drouvot, H. et A.P. Neto (1982), «La dialectique conflit-coopération dans le développement d'une joint-venture : le cas Fibresil », Cahier de Recherche CERAG, $\mathrm{n}^{\circ}$ 82/04, Université de Grenoble, p. 1-17.

DuBISSON, M. (1989), Les accords de coopération dans le commerce international, Paris, Éd. Lamy.

DURAND, C. et al. (1994), La coopération technologique internationale, Bruxelles, Éd. De Boeck.

ERNST, D. et D. O'CONNOR (1989), Technologie et compétition mondiale, un défi pour les nouvelles économies industrialisées, Paris, OCDE.

EVERAER, C. (1993), « Des coûts aux investissements de transaction. Pour un renversement de la théorie de Williamson », Revue Française d'Économie, vol. VIII, $\mathrm{n}^{\circ} 3$, p. 149-203.

GHERZOULI, K. (1995), «Partenariat interentreprises Nord-Sud : Structures explicatives et conditions d'émergence de relations coopératives et réussies », Thèse de doctorat, Université de Bourgogne.

HAMEL, G. (1991), «Competition for competence and interpartner learning within international strategic alliances », Strategic Management Journal, vol. 12, Éd. spéciale, p. 83-103.

HARRIGAN, K.R. (1988), «Strategic alliances and partners assymetries », dans Management International Review, Édition spéciale, p. 53-72.

INGHAM, M. (1990), «Approche stratégique des alliances internationales », Gestion $2000, n^{0} 5$, p. 97-129.

LALl, S. (1990), Promouvoir la compétitivité industrielle dans les pays en développement, Paris, OCDE.

MAPPA, S. (1991), Ambitions et illusions de la coopération Nord-Sud : Lomé IV, Paris, L'Harmattan.

OMAN, C. (1984), Les nouvelles formes d'investissement dans les industries des pays en développement, Paris, OCDE.

OMAN, C. (1989), Les nouvelles formes d'investissement dans les industries des pays en développement, Paris, OCDE. 
Ozawa, T. (1989), Le recyclage des excédents Japonais dans les pays en développement, Paris, OCDE.

POIRIER, L. (1987), Stratégie théorique, vol. II, Paris, Economica.

SCHILlACI, C.E. (1987), « Designing successful joint ventures », Journal of Business Strategy, no 2, p. 59-63.

SNOY, B. (1988), «Entreprises conjointes et coopération Nord-Sud, expérience de la banque mondiale », dans Coopération entre entreprises, ouvrage coordonné par A. Jacquemin, Bruxelles, Éd. De Boeck, p. 140-159.

THOMAS, K.W. (1975), « Conflict and conflict management », dans M.D. Dunette (éd.), Handbook of Industrial and Organizational Psychology, Chicago, Rand McNally.

URBAN, S. et S. VENDEMINI (1994), Alliances stratégiques coopératives européennes, Bruxelles, Éd. De Boeck.

Williamson, O.E. (1975), Markets and Hierarchies: An Analysis and Antitrust Implications, New York, Free Press.

Williamson, O.E. (1985), The Economic Institutions of Capitalism, New York, Free Press.

WOLFE, S. (1995), «Accord inter-entreprises, apprentissage et flexibilité dans le secteur des télécommunications ", dans Coordination Économique et Apprentissage des Firmes, ouvrage coordonné par N. Lazaric et J-M. Monnier, Paris, Economica. 